\title{
Approaches that will yield greater success when implementing self-administered electronic data capture ICT systems in the developing world with an illiterate or semi-literate population
}

\author{
Stanley J. Mierzwa \\ Population Council \\ Samir Souidi \\ Population Council \\ Irene Friedland \\ Population Council \\ Sarah A. Littlefield \\ Population Council \\ Lauren L. Katzen \\ Population Council
}

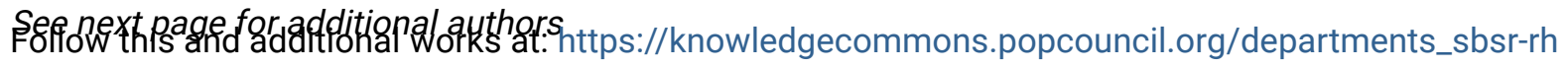

Part of the Community-Based Research Commons, Computer and Systems Architecture Commons, Health Information Technology Commons, and the Health Services Research Commons How does access to this work benefit you? Let us know!

\section{Recommended Citation}

Mierzwa, Stanley J., Samir Souidi, Irene Friedland, Sarah A. Littlefield, Lauren L. Katzen, Craig Savel, Deborah Boccio, and Saumya RamaRao. 2013. "Approaches that will yield greater success when implementing self-administered electronic data capture ICT systems in the developing world with an illiterate or semi-literate population." New York: Population Council. 


\section{Authors}

Stanley J. Mierzwa, Samir Souidi, Irene Friedland, Sarah A. Littlefield, Lauren L. Katzen, Craig Savel, Deborah Boccio, and Saumya RamaRao 
APPROACHES THAT WILL YIELD GREATER SUCCESS WHEN IMPLEMENTING SELF-ADMINISTERED ELECTRONIC DATA CAPTURE ICT SYSTEMS IN THE DEVELOPING WORLD WITH AN ILLITERATE OR SEMI-LITERATE POPULATION

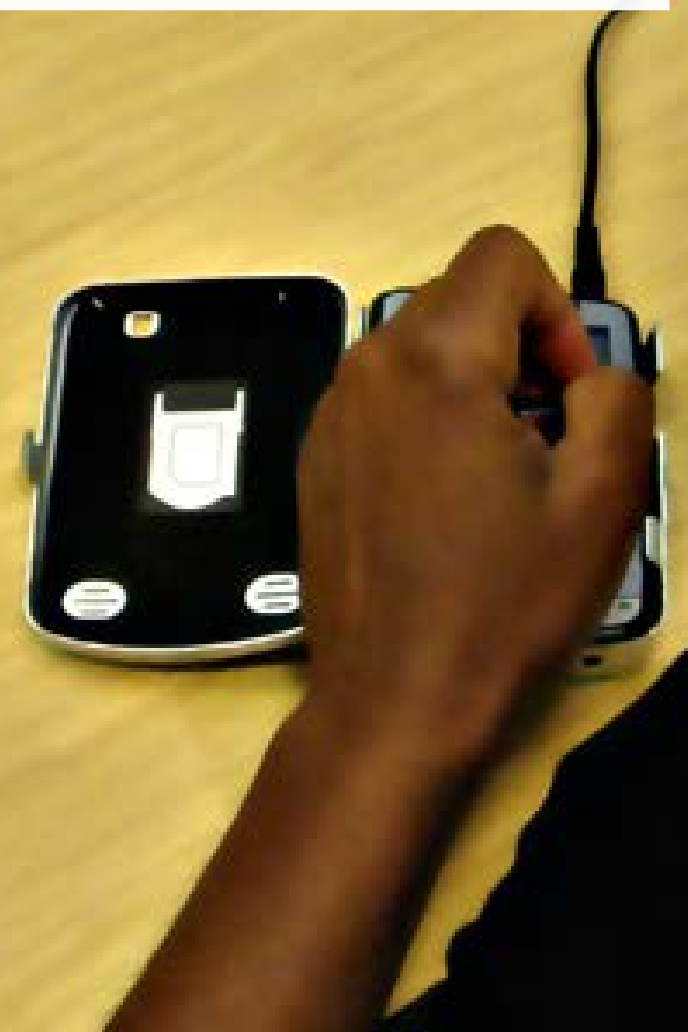

(1) Population Council 
The Population Council confronts critical health and development issues-from stopping the spread of HIV to improving reproductive health and ensuring that young people lead full and productive lives. Through biomedical, social science, and public health research in 50 countries, we work with our partners to deliver solutions that lead to more effective policies, programs, and technologies that improve lives around the world. Established in 1952 and headquartered in New York, the Council is a nongovernmental, nonprofit organization governed by an international board of trustees.

Population Council

One Dag Hammarskjold Plaza

New York, NY 10017 USA

www.popcouncil.org

For more information about this publication, contact the Population Council's Office of Information Technology:

support@popcouncil.org 
Approaches that will yield greater success when implementing self-administered electronic data capture ICT systems in the developing world with an illiterate or semi-literate population

Stan Mierzwa, Samir Souidi, Irene Friedland, Sarah Littlefield, Lauren Katzen, Craig Savel, Deborah Boccio, Saumya Ramarao 


\section{Main Objectives}

Organizations that conduct clinical trials or field- and household-based surveys in the developing world may need to obtain data electronically, especially when attempting to obtain honest and accurate responses to self-administered questionnaires. Successful implementation of information and communication technology (ICT) for self-reporting systems is possible in resourcepoor settings. The objectives of this paper are to discuss the following:

- Field-proven tools and strategies

- Addressing and overcoming implementation issues

- Suggested methods for improving implementation outcomes

- Suggested methods for involving stakeholders

- Benefits of electronic self-administered data capture

- Improvements that can be made by introducing various ICTs

- Examples of ICT in the field (including sample screenshots)

- The future of conducting studies using ICT 


\section{Introduction}

Several years ago, the Information Technology Group at the Population Council responded to a need for a technology solution that would help researchers obtain more accurate responses to survey questions that concerned subject matter of a sensitive nature. The IT group had three goals: (1) to enhance the research by obtaining the best data possible, (2) to operate successfully in the local environment and be welcomed by the local populations, and (3) to increase the efficiency and cost-effectiveness of the survey process.

The IT group built a software/hardware combination solution that met the needs of the researchers and was also accepted by the individuals using the system-an illiterate or semi-literate population that had never seen, let alone used, a computer. The solution involved the development of a fully customized ACASI (audio computer-assisted self-interviewing) module, which is an effective technology for obtaining more honest responses to sensitive questions than the responses received to questions asked face-to-face (Hewett, Mensch, and Erulkar 2004). With ACASI, respondents listen to prerecorded audio questions through headphones connected to a handheld computer and record their responses using a touchscreen. Literate respondents can also simultaneously read the questions on the computer screen. Using ACASI and other ICT solutions allows for standardization, confidentiality, minimized bias, and a more effective collection of survey data.

This paper outlines approaches to consider when designing and implementing self-administered ICT questionnaires in developing countries and reviews the problems and issues we encountered and how we overcame them. Many of the approaches outlined may also be used in general for ICT-related projects in the developing world. 


\section{ACASI Solutions Used in Clinical and Nonclinical Surveys}

The Population Council's customized ACASI module has been used almost exclusively in quantitative research. The following projects used the ACASI solution to survey illiterate or semi-literate populations speaking 21 languages.

\section{Clinic-based studies}

Carraguard $^{\circledR}$ Phase III Trial

Nestorone ${ }^{\circledR} /$ ethinyl estradiol contraceptive vaginal ring

Microbicide Trials Network (MTN)
MTN-035B
MTN-003
MTN-005
MTN-009
MTN-020

Assessing the reporting of sensitive behaviors in microbicide trials

A simulated clinical trial to to explore willingness to participate

\section{South Africa}

Australia, Brazil, Dominican Republic, United

Malawi

South Africa, Uganda, Zimbabwe

India, United States

South Africa

In scale-up: Malawi, South Africa, Uganda, Zambia, Zimbabwe

South Africa

India

1 clinic, 3 satellite sites
2 clinics

14 clinics

3 clinics

7 clinics

17 clinics

3 clinics
13 clinics

\section{Nonclinic-based surveys}

$\begin{array}{ll}\begin{array}{l}\text { Malawi Adolescent } \\ \text { Schooling Study }\end{array} & \text { Malawi } \\ \begin{array}{l}\text { Youth reproductive health } \\ \text { research (pilot) }\end{array} & \text { Vietnam } \\ \begin{array}{l}\text { Assessing and improving the } \\ \text { measurement of sexual } \\ \text { behaviors }\end{array} & \text { Multiple countries } \\ \begin{array}{l}\text { Male Circumcision Partnership: } \\ \text { Achieving scale }\end{array} & \text { Swaziland, Zambia } \\ \begin{array}{l}\text { Prevention with Positives (PwP): } \\ \text { A community-based intervention } \\ \text { study }\end{array} & \text { Mombasa, Kenya } \\ \begin{array}{l}\text { Computerized interviewing } \\ \text { validation }\end{array} & \text { Brazil } \\ \begin{array}{l}\text { Assessing and improving the } \\ \text { measurement of sexual behavior }\end{array} & \text { Uganda }\end{array}$




\section{Survey Design}

\section{Selecting Hardware and User Interface}

Select hardware carefully. Find a platform that is appropriate for the specific type of survey. Decisions concerning which hardware to use to capture data should be made carefully, especially when implementing a project in harsh environments (such as field-based surveys in dusty or dirty settings) or in circumstances where equipment is moved frequently or stored without air conditioning. Although it may seem obvious, procuring hardware that can be supported by local collaborators is crucial. For example, selecting a cutting-edge hardware platform that requires advanced technical training may not be practical.

Choose a touchscreen and stylus. Collecting self-reported data from illiterate or semi-literate respondents has been possible using touchscreen or tablet-based computers and personal digital assistants (PDAs) (Hewett, Mensch, and Erulkar 2004; Gorbach et al. 2012). Regardless of literacy level, many respondents in developing countries have minimal or no experience with computers. For example, some users found it challenging to use a mouse to key in responses. During pretesting and training at a site in Lusaka, Zambia, for MTN-003, we gave users the option of using a mouse, a stylus, or their finger to key in responses. We learned that participants were most successful and comfortable using a stylus. Adoption of a touchscreen design was also found to be essential in an earlier study of self-reporting technology design in the United States (Basch et al. 2007). Therefore, depending on the study design, consider providing additional training if you choose more sophisticated hardware.

Use color-coded keys. In another example of hardware selection, when the Population Council implemented its Carraguard ${ }^{\circledast}$ and Nestorone ${ }^{\circledast} /$ ethinyl estradiol contraceptive vaginal ring clinical trials, touchscreen tablet-based computers were not as prevalent as they are today. Accordingly, simple external number keypads were attached to laptop computers, and color-coded tape was fitted on the keypads to aid participants through the surveys. This was an easy, inexpensive solution to enable illiterate participants to use a computer without having to navigate a keyboard.

Use one computer/PDA model. In addition to hardware selection, adopt one computer/PDA model. This has several advantages, including the ability to standardize documentation and training and to interchange parts if needed. 


\section{Integrating Software and Hardware}

Select and install software matched to the hardware. Our customized ACASI solution is driven by self-contained software that can be installed on a variety of hardware platforms, including computer systems at the sites where the electronic data are collected. This capability reduces the need for local servers. To minimize the problems that could occur when implementing an ICT solution on unknown hardware, provide computer systems with the software already loaded and configured for use, and allow for adjustments needed to tailor the solution to specific users and environments. While dedicated equipment may incur additional charges, we have found that the investment is worthwhile, because it would cost more to ensure that our solution worked on equipment assigned for other tasks and using a variety of hardware, operating systems, or software that could conflict with our software.

\section{Designing the User Interface}

Design simple touchscreens. When providing a technology solution for a semiliterate or illiterate population, ensure that the participants in the survey can easily use the system. Using a combination of audio and graphics is a proven way to capture self-reported data. Keep in mind that too much text and too many icons and interfaces can be confusing and intimidating. A key principle is to keep the screen design simple; using such elements as color-coded buttons or shapes, simple graphics, and number or dial pads to allow participants to answer questions without needing to read; and making sure only one question appears on each screen.

This simple design was also found to be essential in a US-based evaluation (Basch et al. 2007). We provided an audio version of each question in the user's language and asked participants to use a touchscreen system to key in responses. The ability to navigate a screen regardless of a user's level of literacy requires graphic controls that allow replaying a question, going back to the previous question, and proceeding to the next question.

Collaborate with local populations on the graphics. Work with local collaborators to create or review the electronic survey's graphics to ensure cultural appropriateness. For a clinical trial in Nellore, India [MTN-005], we initially created a graphic depicting a couple engaged in sexual relations to be used in questions related to sex acts. Local collaborators suggested changes, such as covering the couple with a blanket for discretion. We used the services of a local illustrator to ensure that all images were consistent and culturally appropriate. This strategy also fared well in earlier research, in cases where handdrawn representations with audio were most accurately recognized (Medhi, Prasad, and Toyama 2007). 
Use graphics to overcome inability to read numbers. We learned another important lesson during the pretest and training for an MTN-005 study in Pune, India, in which one of the questions required recognition of Hindu-Arabic numbers. The number choices were arranged in the shape of a phone dial pad, and participants were required to respond to a question by tapping one or more of the numbers. When a local collaborator commented that approximately 30 percent of the participants would not be able to recognize the numbers, we redesigned the screens to include graphics, rather than relying on a numeric dial pad. We recommend consulting literacy maps and working closely with local collaborators when considering whether to introduce certain types of self-administered questions.

Use culturally-appropriate colors. In another example, during implementation of an ACASI solution in South Africa, we introduced a question type that used color-coded boxes, including blue and green. During pretesting and training at the sites, we were informed that the colors green and blue can be used interchangeably in the local languages and could cause confusion. In this situation, having a system that permitted us to customize and adjust the colors proved instrumental in correcting a problem that could have produced flawed survey results. The experience also helped us realize that, prior to implementation, local staff should confirm that any colors used in the survey are correctly translated into the local language.

Use graphics to indicate lulls in the survey. In implementing combined audio with customized screens, sometimes during the flow of the questionnaire an audio script introduces a series of questions to help outline the series of questions ahead. This is common when new sections are introduced. During the introductions used in a simulated clinical trial [MTN-003], participants thought something was wrong because the screen was blank. To resolve this problem, we displayed a simple graphic in the center of the screens to give participants a visible sign, while they listened to the audio, indicating that the survey was progressing. Similarly, we also added a graphic to the audio script that introduces the study and explains how to manipulate the screens. Figure 1 presents sample buttons that are available on each screen. This method has been reused in numerous studies involving electronic self-reporting and has become part of our standard ACASI solution. 
FIGURE 1. Customized screens and buttons.
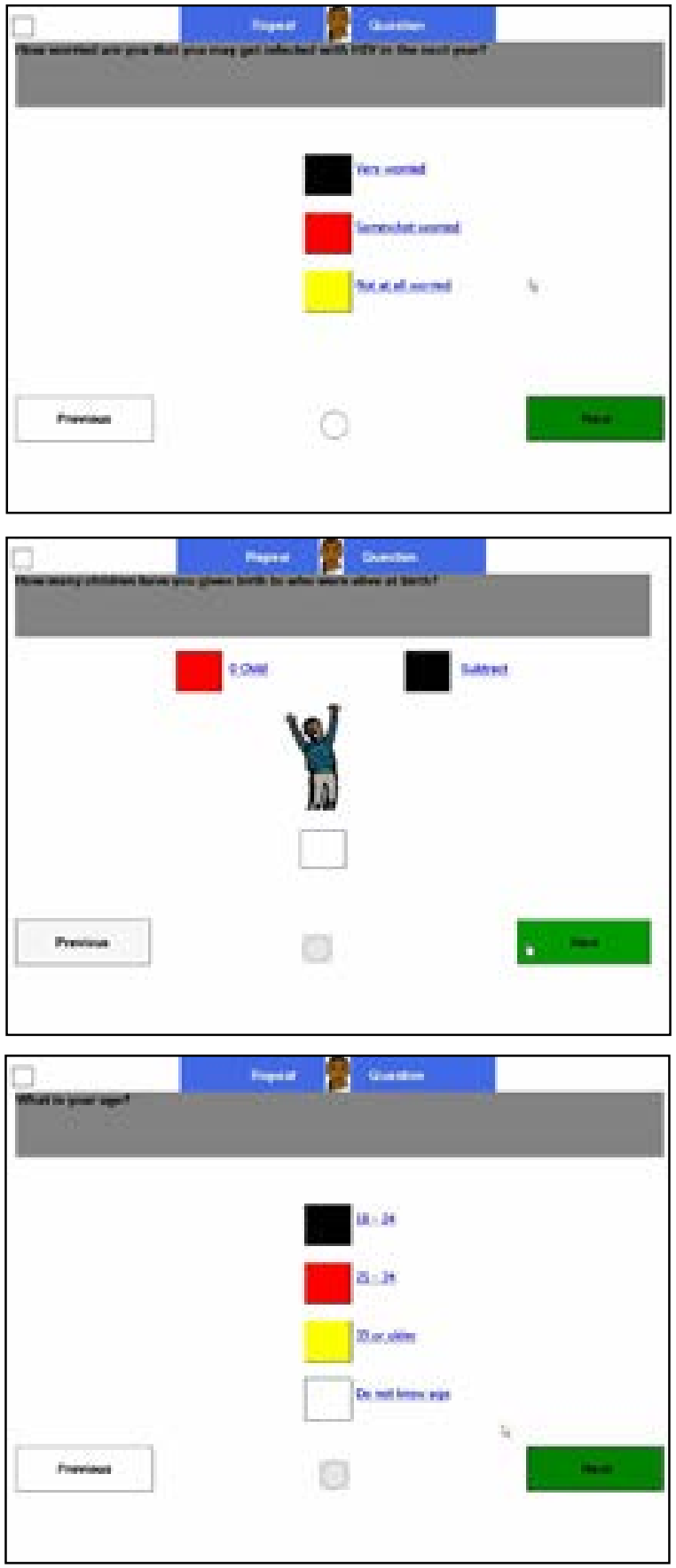


\section{Translating Scripts and Participants' Responses}

Create a translation/back-translation process. We have implemented ICT and ACASI systems in 21 languages. However, as a US-based research organization, the Population Council typically creates the study questionnaire (what appears on the screen) and the accompanying audio script (what participants hear) in English. Furthermore, the majority of our IT staff does not speak the languages spoken by local staff and participants. Accordingly, a well-organized and -documented translation process is essential. Technically, our ACASI system can accommodate numerous languages in one questionnaire setup. So, for example, with an English-based version of the setup, we can add several languages. In some cases, we have programmed for ten different languages in one survey.

Involve local translators and collaborators. Generally, our translation process has included sending English questionnaires and audio scripts to a local translator for translation into the local language(s); a second translator "back translates" the responses into English. The back translation should allow English-speaking researchers to find potential errors in the translation; errors are often attributed to the use of nonresearcher translators or the use of colloquial or complex English terms.

After using this translation/back-translation process for multiple studies, we found that involving local collaborators is essential. For example, participants in one study were asked to rate their ability to adhere to the dosing regimen. Response options ranged from "very poor" to "excellent." During a review of the documents, the IT team recognized that the word used for "poor" was related to poverty instead of inferiority. For some languages, we have also found that a word that might be appropriate to use for the onscreen text would sound awkward if used in the audio script.

Accordingly, a more refined translation process would seek to involve local bilingual and multilingual collaborators during the development of the English questionnaires and audio scripts. Local collaborators who were involved in the development of the English questionnaires should then review the translated documents. Once they are satisfied with the translation, the questionnaire should then be back-translated for US-based researcher review. If time and resources permit, alternatively the translation and back-translation documents might be reviewed by a team. By having multiple US-based researchers, local bilingual and multilingual collaborators, and translators sit together, clarifications can be made about the intended meaning of a question, and the group can come to a consensus about how to adjust problematic questions. Finally, all content, especially content that will appear on the screen, should be carefully proofread. 


\section{Recording Audio Scripts}

Results in a study of optimal audiovisual interfaces for illiterate users of computers demonstrated that audio or voice annotation generally increases the speed of comprehension and accuracy, and decreases the average time taken to complete tasks on the computer (Medhi, Prasad, and Toyama 2007).

Record in a quiet location. Finding a quiet location for recording may be a challenge if recording occurs at busy sites with staff coming in and out of rooms, heavy auto traffic, or other sounds. It can be especially challenging when recording takes place in warmer climates when windows remain open for ventilation or air conditioning units create additional background noise. Identifying a remote or private room well in advance of recording sessions is key. We have found that it is often easiest to record off site. Alternatively, simply posting a "Do not disturb" sign on the door, indicating that recording is in progress, significantly reduced interruptions resulting in the need to rerecord files.

Use a high-quality headset. When creating audio files, it is important to provide a high-quality headset that includes a filtered microphone, usually with a foam cover, which helps to eliminate unwanted ambient sound.

Use standard audio technology. Standard Windows and Apple-based computers provide utilities in the operating system to allow for recording in either 8-bit or 16-bit audio. These utilities can generate .WAV, .MP3, .MP4, or .WMA files. If a utility is not available in the operating system, use free or open-source software options, such as Audacity.

Consider gender when selecting script readers. Because of the sensitive nature of many questionnaires, to enhance participants' comfort we have found it helpful for audio files to be recorded by a person of the same gender as the study participants (i.e., a male voice for male participants, and a female voice for female participants). Furthermore, audio files should be recorded with a local person or staff member (i.e., with a locally recognized accent).

Rehearse script reading. Voice actors should practice reading the script to become comfortable with the source material and flow. On scripts, include tips and visual cues, such as underlining text that needs to be emphasized or indicating when it is important to pause for two to three seconds between phrases. The need to re-record audio files because of interruptions or pauses is common; thus, allow sufficient time to create a quality recording.

Obtain official signoff. Prior to the implementation of any study, ask local collaborators to sign off on the final translated onscreen text, audio scripts, and audio files. 


\section{FIGURE 2. Sample data flow diagram}

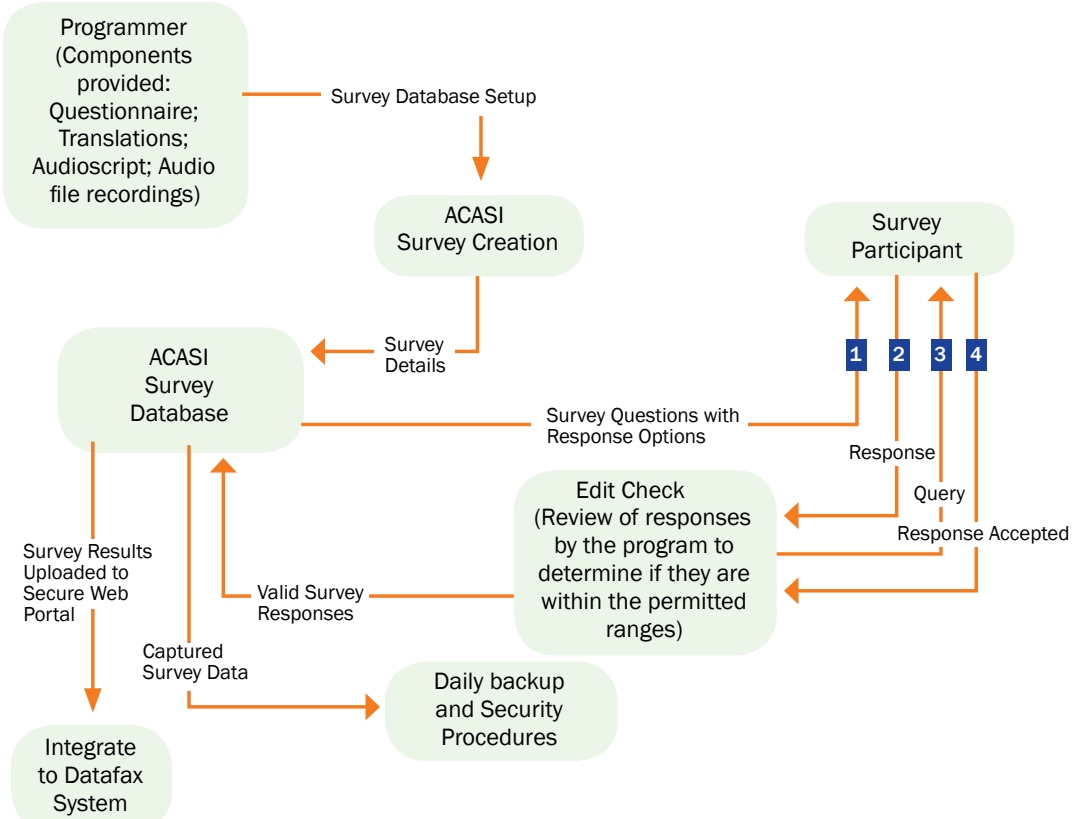

Invite feedback from data analysts. It was important to consult with the data analysts for our database design and to review the data dictionaries to eliminate or minimize problems that could arise when the analysis began. In addition, we provided the data analysts with sample instruments or computers that would be used for the studies to ensure that they understood how the data were being collected and to allow them to offer feedback and suggestions.

\section{Mapping the Flow of the Data}

When collecting self-administered data, map out the flow of the data so that all stakeholders have a pictorial reference to help acquaint them with the process. In many of the projects where we provided ICT systems, parties from several different organizations were involved. In some instances, the resultant data were transferred to another organization that specialized in data analysis and quality control. 
Create a data flow diagram. The idea of creating a data flow diagram occurred to us while we were collaborating on the MTN-035B protocol (Gorbach et al. 2012), in which data were collected to assess participants' adherence in a microbicide gel and condom clinical trial. Collaborators included people from different disciplines (behavioral science, clinical trial management, information technology, statistical science) and organizations (FHI 360, field clinic sites, Magee-Women's Hospital, Population Council, Statistical Center for HIV/ AIDS Research \& Prevention, University of California Los Angeles).

Good documentation is especially useful where local staff will implement the ICT system. A good data flow diagram (Figure 2) (1) allows researchers, information technology staff, and data analysts to get a high-level view of the data capture process; (2) helps staff at the sites understand the key components involved in performing the full-cycle data capture; and (3) is helpful during data analysis and manuscript or report preparation.

Data management can take on different forms. The process described in Figure 2 is a formal arrangement, where different organizations are involved in different aspects of the data flow process. In other instances, such as our longitudinal study of adolescents in Malawi, the data were captured, merged onto the source "Data Manager" computer (i.e., not on the device that collected the original survey results), and emailed weekly (password protected and encrypted) to a designated researcher. This less formal strategy nonetheless ensures that the research scientists receive the data. The process can occur more frequently than weekly if the study protocol requires. 


\title{
Survey Implementation
}

\author{
Ensuring Usability of Hardware
}

Protect the equipment. Using traditional computer equipment for field-based surveys can be particularly challenging. With the innovations in smartphones and PDAs, this is becoming less of an issue, because they are smaller, more mobile, and easier to use. Nevertheless, strategies are needed for protecting the equipment. During the implementation of PDAs for the field-based computer-assisted personal interviewing (CAPI) and ACASI data-collection system of an adolescent survey in Malawi, we procured protective aluminum cases for each of the hardware devices. The cases successfully protected the hardware from damage, resulting in few devices becoming unusable during the five years of use. Such cases are particularly helpful for protecting touchscreen mobile devices.

Provide extra devices. Providing one or more spare devices to sites or interviewers is highly recommended in the event of hardware failure or an increase in sample size.

\section{Planning for Interruptions in Electricity}

Provide batteries and recharging devices. Interrupted electricity is quite common in many of the developing countries where we have provided ICT systems. For this reason, we try to use hardware that permits operation with integrated batteries. Earlier research demonstrated the need to ensure that alternate power was available in the event of power outages (Rotich et al. 2003). Longerlife batteries for laptops, PDAs, and tablets are becoming more readily available and are especially useful during power outages. If the area where the computerized interviews will take place is known to have long power outages or if interviews are being conducted in the field, we make sure spare charged batteries are available. By allowing the hardware device to go into "hibernate mode" (battery drains to a level that requires device shutdown), the battery can be swapped out and the device will boot back up to the place where the electronic survey resumes. When conducting field- or household-based surveys where devices might be without power for days, supplying extra batteries helps but it is also advantageous to provide another means to charge the batteries, such as via car batteries or solar chargers. 
Recharge batteries routinely. An important step to ensure that interviewers are prepared each day with the necessary equipment is to provide an endof-day task list that includes recharging batteries. Wireless electricity could potentially be helpful in combating the issue of interrupted power in remote locations of Africa (IEEE Computer Society 2010).

\section{Managing Data}

Establish quality control and reporting protocols. Part of the task of data management involves doing quality-control checks and reporting back to the datacollection sites when anomalies or issues arise. Monitor or correct key field data on the source Data Manager computer-not on the device that collected the original survey results.

Store data centrally. Implementing self-administered questionnaires requires that the data be stored centrally, on several different levels, to protect the data during collection and to ensure the data can be analyzed frequently. During our project work in collaboration with a trials network, we created a method for structured or tiered data management. This included the concept of the "source" data. Although the data were collected on either PDAs or touchscreen computers, they were merged with a Data Manager system, which was considered to be the source system. Data collected on the Data Manager system should be uploaded to a secure Web portal provided by the organization tasked with monitoring and analyzing the survey results.

\section{Ensuring Data Security}

Security in information technology includes many components. In our studies, and for the purpose of this paper, we focus on three areas: threats from exposure to the internet, keeping data contents secure and private, and security during data transmission.

Protect against malware. Any exposure to the internet creates vulnerability. To help limit exposure to internet threats, in many situations we have asked research and clinic sites to ensure that devices used for computerized interviews not be connected to the internet, except for remote-control support sessions when troubleshooting or updating of systems is required. For the devices we do connect to the internet, we have employed the standard safeguards, such as antivirus, firewall, and anti-spyware software.

Protect passwords and encrypt data. Survey data need to be kept confidential, to protect participants' privacy, to prevent tampering with the data, and to comply with federal regulations. Protecting passwords and encrypting data 
are the minimum steps we have taken with our electronic capture systems. All equipment utilized for our ACASI studies is password-protected, using both power-on and operating system passwords. As an added level of security, the compressed data uploaded from the survey site are also encrypted and password-protected.

Secure data during transmission. We provide ACASI electronic data capture in collaboration with a trials network. The survey result data are uploaded or transmitted from the source computer at the sites to a web-based portal located at a third party's offices and data center. The portal is SSL (secure sockets layer)-encrypted, meaning that the data transmitted from the sites are encrypted and do not flow over the internet in free (i.e., unencrypted) text.

Configure devices to run only the specified software. In most cases, the devices used for trials are preconfigured to run only the ACASI or CAPI software upon boot-up, which eliminates access to other applications.

Lock up devices daily. The final item on interviewers' and site staff's task list is to lock up their electronic devices in a drawer, cabinet, or room at the end of each day.

\section{Backing Up and Storing Data Securely}

Protect data against interruptions in internet access. Many new technologies have become available with the introduction of smartphones. While these devices can deploy data capture systems via the internet, access to the internet may not always be available or may be interrupted, leading to potential loss of data. Similarly, if access is available, internet speeds may not be adequate for good or consistent interaction, which is especially common in remote locations. In this case, "online" is considered any method-mobile broadband, wired, wireless, etc.-that provides connectivity to the internet. Allowing data to be captured regardless of internet connectivity, and securely storing data locally and then uploading or merging to a central source when access is available keeps the system operating and ensures that critical data are not lost.

Do not rely completely on internet connectivity. When collecting data in support of clinical trials, it is imperative that the survey system not rely completely on internet connectivity. Instead, the system must allow the data to be uploaded to a centralized server or portal when internet connectivity becomes available. This model for data flow has worked well in developing countries where internet service is often unreliable. For example, during the training and implementation of an ACASI system in Harare, Zimbabwe [MTN-003], only 
one of three sites had internet connectivity. Being able to store the data securely until a Data Manager computer was available was critical and allowed data collection to take place regardless of connectivity. Additional steps were required to ensure that data from the two nonconnected sites were combined with the main source data at the site, and then ultimately transmitted off-site to a web-based portal. We provided protective cases for the touchscreen computers and had them transported by car to the web-connected site daily or when electronic data capture took place.

Provide a simple utility for daily backups. As with any type of data-collection or electronic system, a simple method for backing up data is essential. Today's backup technology distinguishes between disaster recovery and continuity of data. Disaster recovery allows for restoring lost or technically compromised data, whereas continuity permits the data collection or electronic operation to continue in the event of a major disaster. At a minimum, you should be able to restore data or have the data available off site to protect your data-collection investment.

A simple practice we followed was to provide site staff members responsible for data collection and management with a simple utility (i.e., a Data Manager computer) to do local backups daily. The utility backs up only the data collected and includes all merged survey data that was captured cumulatively. Backing up only the data versus the entire data-collection instrument allowed us to use very simple, inexpensive USB (universal serial bus) memory devices to store backup data securely. Also, we captured and stored images of computer configurations; if an entire computer needed to be restored, this allowed us to quickly build a unit and then restore the data from the USB key.

Provide system redundancy. In addition to local backup, on any day surveys were completed, the resultant data were uploaded or moved off-site to a secure web or FTP (file transfer protocol) site. In addition to backups, we recommend having duplicate survey hardware devices available for site staff who administer electronic surveys.

Capture all data, including incomplete survey data. In all studies or projects that included our ACASI solution, investigators asked us to capture all data, regardless of whether a survey was completed. In response to these requests, we stored the data collected for every survey question. If the system was exited or a survey was terminated before being completed, the data were then saved to the SD (secure digital) card on the PDA or touchscreen tablet computer; in this way, we capured incomplete data as well. 


\section{Assessing Data Quality Regularly}

Assign a user ID to each interviewer. Data quality should be assessed on an ongoing basis. Sometimes, through this process, a particular survey response or scenario needs to be investigated. To aid this undertaking, we recommend assigning each interviewer a user identification (ID), and storing this ID in the survey results database, regardless of whether the survey is completed.

\section{Protecting the Confidentiality of Electronic Data Capture}

Assign participant IDs and store survey responses numerically. An important aspect of our ACASI solution is ensuring the confidentiality of the data collected about participants. Our solution's design assigns numeric values to participants, sometimes called participant identification numbers (PTIDs) or IDs. Inside the results database, no connection is ever made between the PTID or ID and the participant's name or description. In addition, the survey results for each possible question posted to a participant are stored in a numeric value-again, not connected to an actual text value. Typically, the ACASI questionnaires and graphics to be used in a survey, if applicable, are submitted to a local collaborator or Institutional Review Board for review and approval.

\section{Technical support and training}

Assign in-country IT staff with specific training. One of the main goals of implementing an electronic data-collection system away from what is considered our "home base" is to enable local staff to resolve as many issues as possible. For this reason, we generally request that an IT staff person from the local site, or from our country office closest to where the electronic surveys will take place, be present during the training sessions on the system. The local IT staff person will understand generally why a particular technical approach is being taken and will be able to troubleshoot issues, especially when time differences between our US headquarters and the local site would delay a response.

Train in-country IT staff to train the data collectors. When conducting training, we ensure that both programmatic staff and IT staff who helped develop the electronic solution attend. Whereas site IT staff have traditionally not helped build the solutions, they are instrumental in participating in the technical training to assist local site staff and in interfacing with the developers on technical issues that may arise during data collection. Even though training is key to successful implementation of these systems, because data collection can take place over long periods of time, technical issues are bound to arise. 
Create a training and reference manual. As with all data-collection systems, we recommend developing a technical manual for study staff, community workers, clinic staff, and local IT staff. This manual is useful for training new staff and as a reference if issues arise. The manual should include step-bystep instructions for using the survey equipment, a list of equipment to be used, contact information, clear graphics displaying all components of the hardware, sample screens, simple "cheat sheets," a training script for interviewers to follow, and a question-by-question outline in case topic questions are unclear to participants. The manual should also include information about how not to use the equipment. For example, we recommend that equipment be dedicated for study use only and not combined with other computing functions. 


\section{Future challenges}

The movement to ICT solutions to enhance or support innovative research has continued to expand since we began developing solutions for technology-driven self-reporting systems. In many less developed areas, mobile broadband use on smartphones and tablet-based computers is increasing, and more stable electricity is becoming available. However, connectivity still presents challenges and needs to be considered when building an ICT solution for use in these areas. Self-reporting systems such as ACASI can be implemented in these locations, but it should not be assumed that what is built far from the local environment where it will be used will work successfully.

A common goal during the implementation of ACASI for our projects has been to ensure that the system can run independently of technical assistance. Working toward a simple, secure, solid system and providing adequate training and documentation has helped to operationalize the effort well. However, a common request is for a mechanism whereby local partners and researchers can create their own self-administered computerized interviews without the need for technical assistance from IT staff members. While minimizing customizations would help make such a system a reality, we have learned that because of the distinct needs of each study, researchers tend to prefer more customized solutions. However, providing the ability to customize a system for a particular research need-including specialized screens, particular unconventional skip logic, and the like-poses a challenge for making a truly "wizard" type of system available. Another request has been to build a solution that would operate completely over the internet and be available on a variety of hardware platforms, including smartphones and tablets.

To meet these requests, we have begun an effort to build a prototype system that would operate on a variety of devices-when connected to the internet or not (securely storing the data when the system is offline)-and provide an administrative application to step through simple screens to build a self-administered questionnaire with the most frequently used functions. 


\section{References}

Basch, E. et al. 2007. "Evaluation of an online platform for cancer patient self-reporting of chemotherapy toxicities," Journal of the American Medical Informatics Association 14: 264-268.

Gorbach, P.M. et al. 2012."Effect of computer-assisted interviewing on self-reported sexual behavior data in a microbicide clinical trial," AIDS and Behavior 17(2): 790-800.

Hewett, P.C., B.S. Mensch, and A.S. Erulkar. 2004. "Consistency in the reporting of sexual behavior by adolescent girls in Kenya: A comparison of interviewing methods," Sexually Transmitted Infections 80(suppl 2): ii43-ii48.

IEEE Computer Society. 2010. "New technique provides energy wirelessly," Computer 43: 16-19.

Medhi, Indrani, Archana Prasad, and Kentaro Toyama. 2007. "Optimal audio-visual representations for illiterate users of computers," WWW 2007/Track: Technology for Developing Regions, proceedings of the 16th international conference on World Wide Web Pages.

Rotich, J.K et al. 2003. "Installing and implementing a computer-based patient record system in sub-Saharan Africa: The Mosoriot Medical Record System," Journal of the American Medical Informatics Association 10: 295-303.

\section{A selection of this content was published in:}

Mierzwa, Stan, Samir Souidi, Irene Friedland, Lauren Katzen, and Sarah Littlefield. 2013. "Effective approaches to user-interface design with ACASI in the developing world," Interactions XX(3): 58-61. 

(P) Population Council www.popcouncil.org 